\title{
The Impact of Channel Randomness on Coverage and Connectivity of Ad Hoc and Sensor Networks
}

\author{
Daniele Miorandi, Eitan Altman, and Giuseppa Alfano
}

\begin{abstract}
In this paper, we first present an analytical procedure for the computation of the node isolation probability and coverage in an ad hoc network in the presence of channel randomness, with applications to shadowing and fading phenomena. These results are used to obtain an estimate of the connectivity features for very dense networks. Stochastic orderings are used to show the beneficial impact of lognormal shadowing and the negative impact of Rayleigh fading. The impact of SIMO/MISO and MIMO schemes is also addressed, showing how channel diversity can be exploited to enhance network coverage and connectivity.
\end{abstract}

Index Terms-Wireless networks, ad hoc networks, connectivity, coverage, random channels, multiantenna systems.

\section{INTRODUCTION}

$\mathbf{O}$ $\mathrm{NE}$ of the research fields which have gained more attention by the scientific community in the last few years is that of self-organizing large-scale wireless networks, variously referred to as multihop, ad hoc or packet radio networks. Slightly aside, a growing interest is deferred to wireless sensor networks [1], which, while presenting peculiar features, possess at the same time many of the characteristics of ad hoc networks.

One of the issues of more concern, for such networks, is that of limiting achievable performance, in terms of capacity, connectivity, delay and coverage [2], [3], [4]. Most of the published works still rely on a simplistic model of channel propagation, where the randomness inherently present in radio communications is not considered. During the last couple of years, however, a growing interest has risen on investigation of channel randomness impact on limiting achievable performance of random networks.

In this work, we characterize the relationship linking, in the presence of a generic random channel, the node isolation probability and the second-order moment of the communication range. This will be approached generalizing a result obtained (implicitly) in [5] for the node isolation probability in the case of lognormal shadowing. We then show that, under our assumptions, the coverage probability, an important

Manuscript received October 17, 2006; revised February 11, 2006; accepted March 28, 2007. The associate editor coordinating the review of this paper and approving it for publication was Q. Zhang. Part of this work has been presented at IEEE INFOCOM 2005. This work has been partially supported by the EU within the framework of the BIONETS project EU-IST-FP6-FETSAC-027748 (www.bionets.eu).

D. Miorandi is with CREATE-NET, Via Solteri 38, 38100, Trento, Italy (e-mail: daniele.miorandi@ create-net.org).

E. Altman is with INRIA, 2004 Route des Lucioles, 06902, Sophia Antipolis, France (e-mail: eitan.altman@sophia.inria.fr)

G. Alfano is with the Univ. of Sannio, p.za Roma, 82100, Benevento, Italy (e-mail: gialfano@unisannio.it).

Digital Object Identifier 10.1109/TWC.2007.060842. performance metric and design parameter in sensor networks, coincides with the complement of the node isolation probability obtained with an "equivalent communication range" having the same distribution of the sensing range. Further, under the assumption of very dense networks (to be formalized later), we provide an estimate of the probability that any finite portion of the network is connected. The possibility of exploiting the channel randomness by means of diversity schemes is also considered, showing that the network connectivity features may be enhanced with the adoption of multiple antennas or equivalent diversity schemes. The analytical results are compared with the outcomes of numerical simulations. It is also analytically proven that techniques based on the random selection of the transmission power (with an average power constraint) cannot enhance the network connectivity with respect to the case in which all nodes transmit at the same power.

The paper is organized as follows. Sec. II presents related work and details the novel results contained in the paper. Sec. III presents the framework and reports the computation of the node isolation probability under the channel models mentioned above. The results are then used in Sec. IV to get an estimate of the connection probability in very dense networks. The impact of diversity schemes is investigated in Sec. V. Sec. VI concludes the paper indicating some directions for future research.

\section{RELATED WORK}

Connectivity and coverage in wireless ad hoc/sensor networks have received considerable attention over the last few years. In [6] the problem of finding a necessary and sufficient scaling for the transmission range to obtain a fully connected network (in the limiting dense regime) was studied. The same results were obtained independently by Penrose in a more abstract framework [7]. Scalings for the case of sparse networks were investigated in [8]. The connectivity achievable in the presence of nodes mobility was first addressed in [9]. All these works rely on a simplistic model of radio waves propagation, where nodes communicate to each other if and only if their distance is smaller than a given value.

The impact of lognormal shadowing on connectivity of ad hoc networks was first addressed by Bettstetter and Hartmann [10] (which extends a previous conference work). In particular, they proposed a numerical method for evaluating the impact of lognormal shadowing. The same issue is dealt with in [11] (which extends a previous conference paper by the same authors), where qualitative results are presented. In [12] we provided a closed-form expression for such case. The same 
result had been (implicitly) obtained by Orris et al. in a different framework (cellular networks) [5]. The same authors also extended their work to the superposition of shadowing and fading phenomena [13], [14]. The impact of Rayleigh fading on network connectivity was studied by Haennggi in [15]. All these results can be seen as particular cases of the general case first analyzed in [12]. In that respect, this paper contains two important generalizations, i.e., to the case of MIMO channels (with maximal ratio combining) and to the case of Rician fading.

\section{ANALYSis of THE NODE ISOLATION PROBABILITY}

\section{A. System Model}

Let $\mathcal{N}$ be a two-dimensional stationary Poisson point process over $\Re^{2}$, defined on a probability space $(\Omega, \mathcal{F}, \mathbb{P})$. We denote by $\mathbb{E}[\cdot]$ the expectation taken with respect to the measure induced by $\mathbb{P}$. The points of the process represent the location of the devices. The process is assumed to have intensity $0<\lambda<+\infty$, where $\lambda$ is defined as the expected number of points in the unit square. Given a bounded subset $A \in \Re^{2}$, the number of points of $\mathcal{N}$ in $A$, denoted by $\mathcal{N}(A)$, is a Poisson random variable of intensity $\lambda \nu(A)$, where $\nu(A)$ is the area of $A$. All devices transmit at a fixed power level $P_{t x}$. We consider as "receiver" an arbitrarily located point, which is independent of the process $\mathcal{N}$. We pose questions concerning one single receiver, but the results apply to any number of receivers provided that interference (created by possibly multiple simultaneous transmissions) is negligible. We assume that a white noise of power $W$ is present at the receiver.

In our study of connectivity, we neglect the impact of interference. Our results are thus valid in the presence of low traffic load or when an efficient MAC layer protocol is present, so that we may disregard the mutual interference generated by the devices. Although this may seem an optimistic assumption (the impact of interference on connectivity is widely discussed in [16], [17]), in our view the connectivity is a "limiting" performance, representing in some sense the supremum of the connectivity properties over the whole range of MAC protocols.

The modeling of propagation in a radio channel is a complex task, which heavily depends on the environment under study (indoor, outdoor urban, outdoor land etc.). The received power is given by the product of $P_{t x}$ and the path loss $l$, which is usually described by means of random variables ${ }^{1}$. The mean of the path loss is given by a law of the type $K r^{-\alpha}$, where $K$ is a constant which depends on some physical layer system features and $r$ represents the transmitter-receiver distance $^{2}$. The path loss exponent, $\alpha$, is usually in the range

\footnotetext{
${ }^{1}$ Note that, according to our definition, the path loss should be understood as "path gain".

${ }^{2}$ As widely discussed in [18], the fact that the path loss goes to $\infty$ when $r$ goes to zero may lead to optimistic conclusions in the analysis of scaling laws for dense networks. However, this phenomenon appears as long as we take interference into account. As stated therein, "For models neglecting interferences $(. .$.$) these changes from a strict power law attenuation function$ may be only second order effects on the performance of the network". Also, in some situations where absorption plays a major role (e.g., underwater communications) the path loss can follow also an exponential decrease.
}

$(2,4)$, while for some particular application scenarios it can be taken as large as 10 [19].

As far as the randomness inherently present in the radio channel is concerned, two different effects are usually considered, which may be observed over different time (or space) scales. The first, referred to as shadowing, reflects the different propagation conditions which may be encountered by radio waves due to buildings, terrain roughness, foliage and other obstacles. In other words, it considers that two terminals, located at the same distance by a transmitter, may experience different received signal power. A widely acknowledged model for shadowing predicts the received power to be lognormally distributed (i.e. the received power, expressed in $\mathrm{dB}$, is normally distributed) [19]. The shadowing phenomena are also referred to as "medium scale" or slow fading, to distinguish them from the "small scale" or fast fading phenomena. The latter terms describe one of the peculiarities of radio wave propagation, i.e. the rapid fluctuation of the amplitude of a radio signal over a short period of time or travel distance. (Since we are considering static networks, the fading has in our case to be understood as a phenomenon which acts over the spatial dimension, even if it could also take place over the time dimension due to reflections from moving objects.) In the presence of dense multipath conditions, each received signal may be modelled as the superposition of two orthogonal normal components, so that the signal envelope is distributed as a Rayleigh random variable. In turn, this leads to an exponential distribution for the received power [20].

Our analysis starts with the computation of the probability of a node being isolated, denoted by $P_{I}$, meaning that none of the other nodes present in the network is able to communicate with it. We denote by $P_{S \mid \Gamma}(y)$ the packet success probability given that the received signal presents an average signal-tonoise ratio (SNR) $\Gamma=y$. In our model, the packet error rate versus instantaneous signal-to-noise ratio is approximated by a step-like function, the threshold being denoted by $\Psi$. Such a model works, e.g., in the case when good long codes are used. (A similar model, called "physical model", has also been used in [3].) The parameter $P_{S \mid \Gamma}(y)$ depends therefore only on the distribution of the instantaneous SNR, given its average value.

In this work, we assume that the fading processes on the various links are independent. While such assumption may not reflect a real network environment (especially as the node density becomes large), it still enables us to obtain some closed-form expressions which may be used as estimates for more realistic situations and compared with testbed measurements. In particular, we could expect that, in the presence of links for which the attenuation is correlated, the connectivity properties of the network will degrade with respect to the independent case. In a sensor network framework, one interesting performance metric is the coverage probability, which plays a fundamental role in intrusion detection and other applications [21].In particular, we focus on networks where sensing relies on wave propagation laws which are those which guide signal propagation in the ad hoc case. In general, coverage is based on the sensing range which is usually different from the communication range. The node isolation probability is the probability that a "typical" node is not connected to any other nodes, i.e. that it is not covered by the 
footprint generated by all other nodes. Let us assume (with a slight abuse of terminology) that in this case the "connectivity" is based not on the communication range but, rather, on the sensing range. On the other hand, the coverage probability $P_{\text {cov }}$ is the probability that a "typical" point is covered. If the point process representing devices location is stationary, we can consider the event $\{$ the origin is covered $\}$. The coverage probability is the probability of such event under the measure induced by $\mathbb{P}$. On the other hand, the node isolation probability is the complementary of the probability of such event under the measure induced by the Palm probability [22] associated with the point process $\mathcal{N}$. Since we assumed $\mathcal{N}$ to be a Poisson process, we can exploit Slivnyak's theorem ${ }^{3}$ [23], obtaining:

$$
P_{\text {cov }}=1-P_{I} \text {. }
$$

In case $\mathcal{N}$ is a more general point process (stationary ergodic), the two quantities can be related by means of a Palm inversion formula [23].

\section{B. A Deterministic Path-Loss Model}

The deterministic case will be used, in the following, as a reference to show the impact of the channel randomness induced by the shadowing and fading phenomena. In the case of a deterministic channel model, there exists a deterministic distance $R$ (referred to, in the following, as the communication range), so that a node is able to communicate with all the nodes lying within distance $R$. The signal-to-noise ratio (SNR) in the presence of a transmitter-receiver distance of $r$ is given by $\gamma(r)=\frac{P_{t x} l(r)}{W}$, where in this case $l(r)=K r^{-\alpha}$. The communication range may be computed as the distance at which the SNR falls below the threshold $\Psi$. Thus, we get:

$$
R=\left(\frac{K P_{t x}}{W \Psi}\right)^{\frac{1}{\alpha}} .
$$

This is indeed a classical Boolean model with deterministic (circular) shapes [24], and the node isolation probability is given by:

$$
P_{I}=e^{-\lambda \pi R^{2}}=e^{-\lambda \pi\left(\frac{K P_{t x}}{W \Psi}\right)^{\frac{2}{\alpha}}} .
$$

\section{The Impact of Channel Randomness}

Let us consider a case where the channel model presents a random component. We assume the path loss, given a transmitter-receiver distance $r$, to be described by a probability density function (pdf) $f_{l \mid r}(\cdot \mid \cdot)$, or, equivalently, by the corresponding cumulative distribution function $F_{l \mid r}(\cdot \mid \cdot)$. We denote by $l_{i}$ the loss on the path between the intended device and node $i$. A node is isolated if, for any $i$, the SNR $\frac{P_{t x} l_{i}}{W}$ falls below the threshold $\Psi$. We define a random variable $R$, which will be referred to as the communication range in the following, having cumulative distribution function:

$$
\begin{aligned}
F_{R}(a)= & \mathbb{P}[\gamma(a) \leq \Psi]=\mathbb{P}\left[l(a) \leq \frac{W \Psi}{P_{t x}}\right]= \\
& =\mathbb{P}\left[l(r) \leq \frac{W \Psi}{P_{t x}} \mid r=a\right]=F_{l \mid r}\left(\frac{W \Psi}{P_{t x}} \mid a\right) .
\end{aligned}
$$

\footnotetext{
${ }^{3}$ Roughly speaking, Slivnyak's theorem states that the distribution of points in a Poisson process does not depend on the assumption of the existence of a point at the origin.
}

In the more general case where $P_{S \mid \Gamma}(\cdot)$ is not a step function, we have:

$$
F_{R}(a)=1-\int_{0}^{+\infty} f_{l \mid r}\left(\frac{W x}{P_{t x}} \mid a\right) P_{S \mid \Gamma}(x) \mathrm{d} x .
$$

Intuitively, the communication range determines the probability that one device is able to communicate to another one located at distance $a$. The deterministic path-loss model can be seen as a special case, having the whole mass concentrated on a single value $R$. We assume $0<\mathbb{E}\left[R^{2}\right]<+\infty$. The cases $\mathbb{E}\left[R^{2}\right]=0$ and $\mathbb{E}\left[R^{2}\right]=+\infty$ are trivial and of no interest: in the first case our device is disconnected $\mathbb{P}$-a.s., whereas in the second one it is connected with all other devices $\mathbb{P}$-a.s. Triviality extends also to network connectivity (see [25] for a percolation argument).

We now compute the distribution of the number of one-hop neighbors of a device, generalizing a result in [5]. The proof can be found in [12].

Theorem 3.1: Given that nodes are distributed according to a Poisson process of intensity $\lambda$, and denoting by $R$ the communication range, the number of one-hop neighbors follows a Poisson law of intensity $\lambda \pi \mathbb{E}\left[R^{2}\right]$.

As a corollary, the node isolation probability is given by:

$$
P_{I}=e^{-\lambda \pi \mathbb{E}\left[R^{2}\right]} \text {. }
$$

Formula (6) is worth some comments. Indeed, it predicts that channel randomness may have a beneficial impact on network connectivity. This holds if and only if the physical phenomenon which induces the randomness leads to an increment in the second-order moment of the random variable $R$.

On the other hand, (6) can also be used in the network dimensioning phase, as we shall do in the following Sections, in that we can choose a given $\lambda_{\varepsilon}$ which ensures that any randomly chosen point in the network is covered with probability $1-\epsilon$ :

$$
\lambda_{\varepsilon}=-\frac{\log \varepsilon}{\mathbb{E}\left[R^{2}\right]} .
$$

\section{Analysis in the Presence of Shadowing}

In [10] a semi-analytical approach is presented for the computation of the node isolation probability in a shadowing environment. In particular, the procedure is based on the distribution of the $k$-th nearest neighbor distances, but no closed-form formula is obtained. By following the procedure outlined in the previous subsection, we are able to get a closedform expression for $P_{I}$, from which it is possible to clearly see the impact of the different factors. Given a transmitter-receiver distance $\rho$, the pdf of the path loss is given by [26]:

$$
f_{l \mid r}(a \mid \rho)=\frac{1}{\sqrt{2 \pi} \sigma a} e^{-\frac{1}{2}\left(\frac{\ln a-\ln (K \rho-\alpha)}{\sigma}\right)^{2}},
$$

where $\sigma$, the lognormal spread, is the standard deviation of the Gaussian distribution describing the shadowing phenomenon. The cumbersome derivation, reported in [12] leads to:

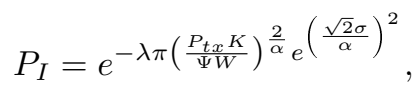

a result that was derived also in [5]. 
This suggests that the presence of lognormal shadowing improves the connectivity properties of the network, as predicted by the results in [11], [27]. Further, the node isolation probability is monotonically decreasing in the lognormal spread $\sigma$. This fact may also be seen as a byproduct of a more general result, which regards the possibility of finding a stochastic ordering ${ }^{4}$ among the distributions of the communication range with different values of the lognormal spread $\sigma$. Indeed, we have:

$$
\begin{aligned}
F_{R_{\sigma}}(a) & =1-\int_{0}^{\frac{\Psi W}{K P_{t x}}} \mathrm{~d} x \frac{1}{\sqrt{2 \pi} \sigma x} e^{-\frac{1}{2}\left(\frac{\ln \left(x a^{\alpha}\right)}{\sigma}\right)^{2}}= \\
& =\int_{-\infty}^{\frac{\ln \frac{\Psi W a^{\alpha}}{K P}}{\sigma}} \frac{\mathrm{d} t}{\sqrt{2 \pi}} e^{-\frac{t^{2}}{2}}=\Phi\left(\frac{\ln \frac{\Psi W a^{\alpha}}{K P_{t x}}}{\sigma}\right) .
\end{aligned}
$$

It is then easy to see that we may write:

$$
R_{\sigma_{1}} \preceq R_{\sigma_{2}}, \quad \sigma_{1} \leq \sigma_{2} .
$$

From [28], (11) is equivalent to $\mathbb{E}\left[f\left(R_{\sigma_{1}}\right)\right] \leq$ $\mathbb{E}\left[f\left(R_{\sigma_{2}}\right)\right], \quad \sigma_{1} \leq \sigma_{2}$ for any increasing function $f(\cdot)$. Hence, we have that:

$$
\mathbb{E}\left[R_{\sigma_{1}}^{h}\right] \leq \mathbb{E}\left[R_{\sigma_{2}}^{h}\right], \quad \sigma_{1} \leq \sigma_{2}, h \geq 0 .
$$

Taking $h=2$ and recalling (6), we retrieve that the node isolation probability decreases monotonically with the lognormal spread $\sigma$.

In the limit $\sigma \rightarrow+\infty$ the behavior of the resulting network will resemble that of an Erdös-Rényi random graph [29], for which the distance between any node pairs has no impact on the probability of having a direct link between such nodes. In such limiting regime, the probability that a typical node is connected to any other node tends to $\frac{1}{2}$. Indeed the probability of connection to a node at distance $\rho$ is:

$$
1-F_{R_{\sigma}}(\rho)=Q\left(\frac{\ln \frac{W \Psi \rho^{\alpha}}{P_{t x} K}}{\sigma}\right),
$$

which for any finite $\rho$ tends to $Q(0)=\frac{1}{2}$ as $\sigma \rightarrow+\infty$. The probability that, given $n$ other nodes in the network, the typical node is isolated is $\frac{1}{2^{n}} \rightarrow 0$ as $n \rightarrow+\infty$. Since in our unbounded region there is $\mathbb{P}$-a.s. an infinite number of nodes, our network will $\mathbb{P}$-a.s. not present any isolated node, which provides an informal justification of the fact that $\lim _{\sigma \rightarrow+\infty} P_{I}=0$. On the other hand, if $\sigma \rightarrow 0$ we retrieve the deterministic path-loss model previously analyzed. It is interesting to note that in such limiting case, the connectivity properties are driven only by the features of the underlying spatial process; in this sense, we may regard the resulting network as a geometric random graph [30] (this relationship between ad hoc networks and geometric random graphs is not new, see [31]). In some sense, the partial randomness introduced by the lognormal shadowing may be expected to produce a network similar to a small world [32], which is indeed known to present better connectivity properties than geometric random graphs.

\footnotetext{
${ }^{4}$ Given two random variables $A$ and $B$, we say that $A$ is stochastically smaller than $B, A \preceq B$ if $F_{A}(x) \geq F_{B}(x) \forall x \geq 0$ [28].
}

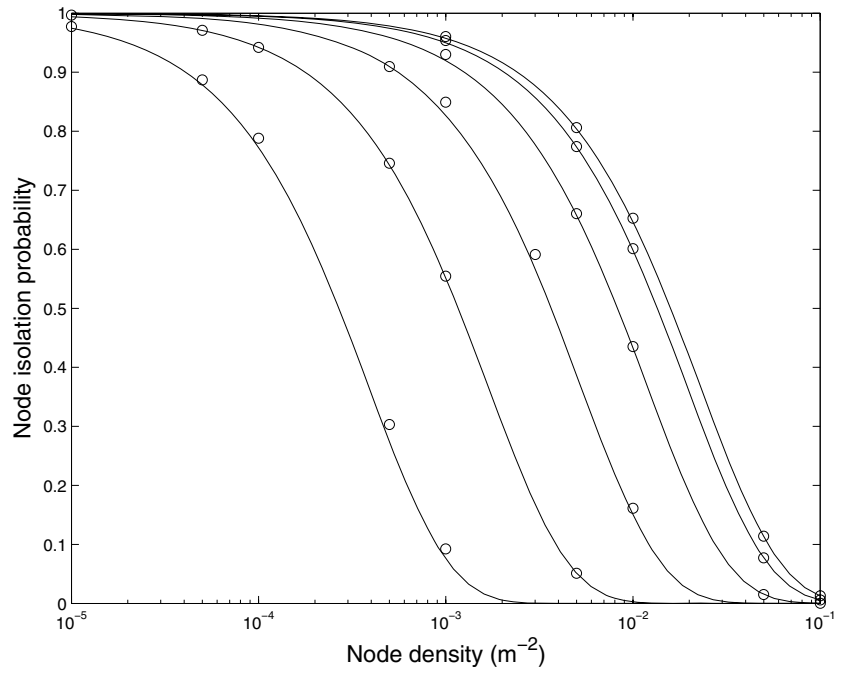

Fig. 1. Node isolation probability vs. node density in the presence of lognormal shadowing, $\alpha=3.5, P_{t x}=1$ mWatt, $K=10, W=0.01$ mWatt, $\Psi=10 \mathrm{~dB}, \sigma=0,1, \ldots, 10$ (a larger value of $\sigma$ corresponds to a curve to the left). Circles represents simulation results (averaged over 1000 simulations)

This, indeed, can be seen noting that as $\rho \rightarrow+\infty$, (13) matches the connection law in [33], hence our model is likely to exhibit long-range connections. Related results are obtained in [34], where the authors, by using a continuum percolation approach, show the beneficial impact of spread-out connections (i.e., a spread-out density for $R$ in our setting) on the network connectivity keeping a fixed $\mathbb{E}\left[R^{2}\right]$. On the other hand, we demonstrated that some forms of channel randomness (e.g., lognormal shadowing) are able to improve connectivity features by actually enlarging $\mathbb{E}\left[R^{2}\right]$ without any increase in $P_{t x}$. The interesting phenomenon observed in [34] impacts the critical density at which network percolation occurs. However, this is not directly related to the node isolation probability, but to its relationship with the probability of existence of an infinite component. Further, in the limit $\lambda \rightarrow+\infty$ the enhancement due to spread-out connections vanishes, since the probability of existence of an infinite component tends to 1 at the same rate as $P_{I}$ (which depends just on $\mathbb{E}\left[R^{2}\right]$ ) tends to 0 [25].

It is worth remarking that these results do not account for the basic fact that a wireless channel, in reality, cannot amplify a signal, so that the impact of lognormal shadowing in a real setting is not completely clear. ${ }^{5}$ Further, notice that, with the lognormal shadowing model considered, we are introducing a bias, in that $\mathbb{E}[R]=\left(\frac{P_{t x} K}{\Psi W}\right)^{\frac{1}{\alpha}} e^{\left(\frac{\sigma}{\sqrt{2} \alpha}\right)^{2}}$, so that the average path gain increases with the lognormal spread.

In Fig. 1 we plotted some results, in terms of node isolation probability versus the node density $\lambda$ for various values of the lognormal spread $\sigma$. The other system parameters are $\alpha=3.5$, $P_{t x}=1$ mWatt, $K=10, W=0.01 \mathrm{mWatt}, \Psi=10 \mathrm{~dB}$. From the curves it is evident that lognormal shadowing may have a tremendous impact on network performance, leading to a large

\footnotetext{
${ }^{5}$ The shadowing is, indeed, caused by obstacles between the transmitter and the receiver, blocking and/or reflecting the useful signal; hence, only in ideal cases pure reflection is allowed, while the overall effect is actually of signal power absorption.
} 


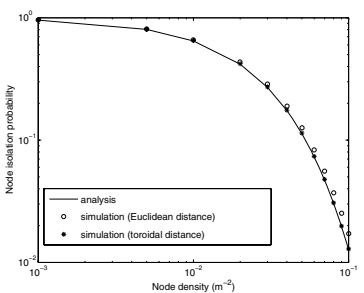

(a) $\sigma=0$

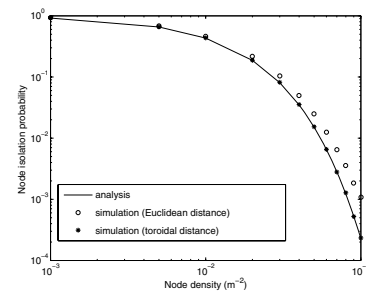

(c) $\sigma=2$

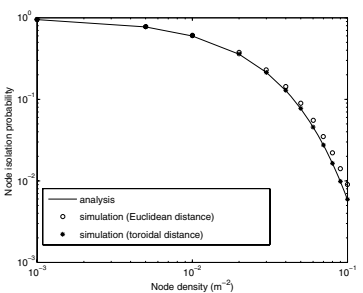

(b) $\sigma=1$

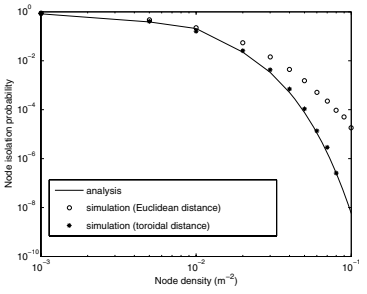

(d) $\sigma=3$
Fig. 2. Node isolation probability for $\alpha=3.5, P_{t x}=1 \mathrm{mWatt}, K=10$, $W=0.01 \mathrm{mWatt}, \Psi=10 \mathrm{~dB}$.

performance enhancement, with respect to the deterministic channel model (correspondent to $\sigma=0$ ), even for low values of the lognormal spread.

In order to validate our results, we simulated a $100 \mathrm{~m} \times$ $100 \mathrm{~m}$ square, and took the same system parameters as above. We considered a node density $\lambda=0.04 \mathrm{~m}^{-2}$ and varied $\sigma$ between 0 (path loss only) and 3 . We then computed the node isolation probability averaging over 1000 simulations. The results are plotted in Fig. 2, where for the simulation results we reported the mean value.

It may be seen that our analysis is able to closely follow the system behavior for a wide range of node density, our analysis slightly overestimating the node isolation probability. This effect is due to the fact that our analysis has been carried out for an unbounded region, neglecting thus the border effects that arise in the presence of a finite area [35]. In such a case, in fact, nodes placed close to the border regions are more likely to be isolated, since they can connect only to devices placed in a region of area smaller than $\pi \mathbb{E}\left[R^{2}\right]$. The border effect, in our example, has an impact which is increasing with an increase in the mean covered area $\pi \mathbb{E}\left[R^{2}\right]$, as it may be seen in Fig. 2.d. This explains the small mismatch between analysis and simulation data. Simulation results obtained through the use of a toroidal distance, which allows to get rid of border effects are plotted as well, showing a better match with theoretical curves.

\section{E. Analysis in the Presence of Superimposed Lognormal Shadowing and Rayleigh Fading}

The analytical procedure showed above may be easily extended to more complex scenarios, such as that where both lognormal shadowing and fast (Rayleigh) fading are simultaneously present [13]. Let $\Gamma$ be the average SNR

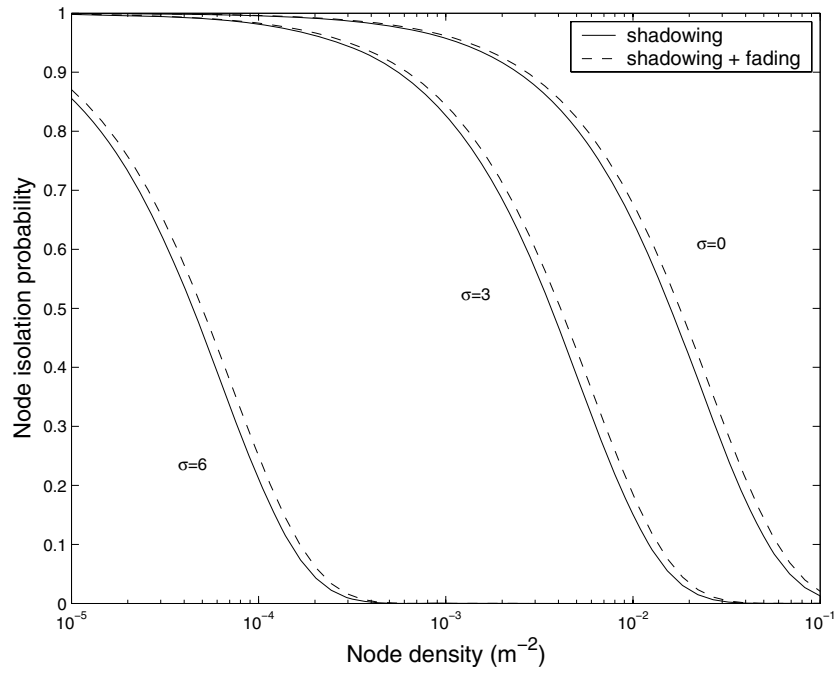

Fig. 3. Node isolation probability vs. node density: the impact of Rayleigh fading; $P_{t x}=1$ mWatt, $K=10, W=0.01$ mWatt, $\Psi=10 \mathrm{~dB}, \alpha=3.5$, $\sigma=0,3,6$ (a larger value of $\sigma$ corresponds to a curve to the left).

(meaning that it is averaged over the variations of the smallscale fading), and consider again a threshold-like success probability, with threshold equal to $\Psi$. The instantaneous SNR $\gamma$ is exponentially distributed with mean $\Gamma=y[20]$ so that we have:

$$
P_{S \mid \Gamma}(y)=\int_{\Psi}^{+\infty} \mathrm{d} a f_{\gamma \mid \Gamma}(a \mid y)=\int_{\Psi}^{+\infty} \mathrm{d} a \frac{1}{y} e^{-\frac{a}{y}}=e^{-\frac{\Psi}{y}} .
$$

Substituting in (6), we obtain, after some algebra [12]:

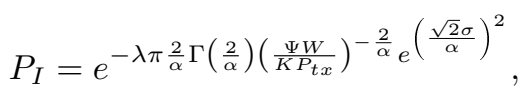

where $\Gamma(\cdot)$ represents the usual Gamma function.

Since $y \Gamma(y) \leq 1$ for any $y \leq 1$, equality holding only for $y=1$, we have that, for $\alpha>2$ (note that the case $\alpha=2$ corresponds to free-space propagation, so that for any real system it is $\alpha>2$ ) Rayleigh fading reduces the connectivity properties of the network. Indeed, also the bias in the average path gain actually reduces in the presence of Rayleigh fading. This can be explained by simply recalling the negative impact of Rayleigh fading on an AWGN wireless link, where transmitted power is isotropically spread causing on average a negative drift of the received power. As for the case of traditional wireless communications on Rayleigh faded links, such a negative impact can be partially avoided by means of diversity, whose exploitation will be investigated in the following sections. This holds for any $\sigma$, so that the negative influence of the Rayleigh fading does not depend on the underlying shadowing phenomena.

In Fig. 3, a comparison between lognormal shadowing and superimposed shadowing and Rayleigh fading is shown. Notice that the Rayleigh fading, although having a negative impact on the connectivity properties of the network, does not change much the node isolation probability. This is further confirmed by Fig. 4 where we plot the required node density to achieve a prescribed node isolation probability according to (7), for two values of the lognormal spread. The curves 


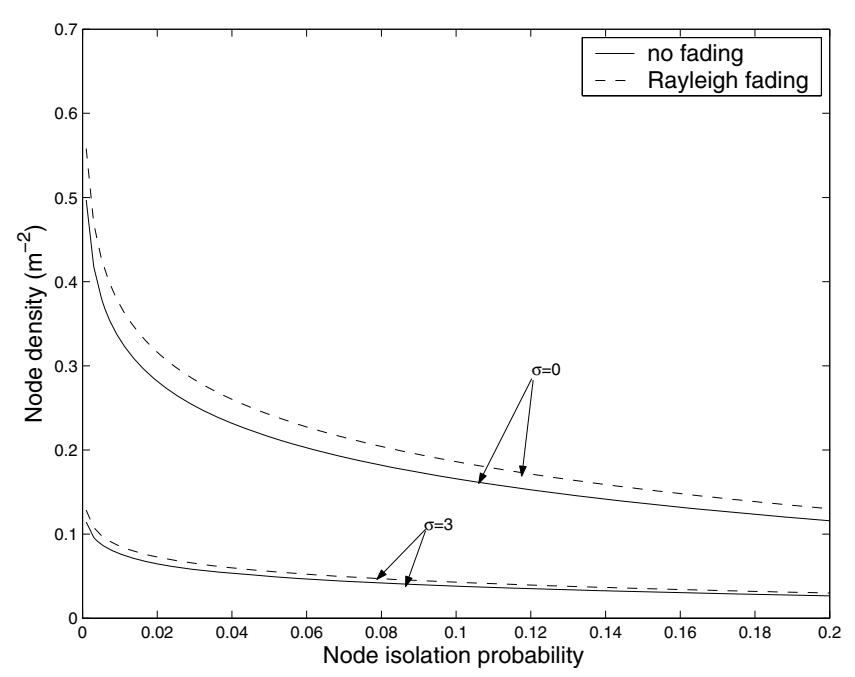

Fig. 4. Node density vs. node isolation probability : the impact of lognormal spread and Rayleigh fading on network dimensioning; $P_{t x}=1 \mathrm{mWatt}, K=$ $10, W=0.01 \mathrm{mWatt}, \Psi=10 \mathrm{~dB}, \alpha=3.5, \sigma=0,3$ (a larger value of $\sigma$ corresponds to a curve to the bottom of the figure).

confirm both the positive impact of lognormal shadowing as well as the slightly negative impact of Rayleigh fading at the network dimensioning stage, since to achieve a prescribed isolation probability a slightly higher node density is required in the case of superimposed Rayleigh fading with respect to the case of lognormal shadowing with no fading. Furthermore, the required node density scales down with increasing values of the lognormal spread.

\section{F. Analysis in the Presence of Rician Fading Channels}

Rayleigh fading models worst-case situations, in which all the received power comes from reflection and scattering. A more realistic alternative model exists, called Rice fading, in which a line-of-sight path is present, and there are also a large number of independent paths. This model is characterized by a parameter $\kappa$, defining the ratio of the energy in the line-ofsight path to the energy in the scattered paths. For the case $\kappa=0$ we return to Rayleigh fading, whereas if $\kappa \rightarrow+\infty$ the model approaches a deterministic channel.

We start by computing the probability of successful reception in the presence of an average $\operatorname{SNR} \Gamma=y$ :

$$
\begin{aligned}
& P_{S \mid \Gamma}(y)=\int_{\Psi}^{\infty} d a f_{\gamma \mid \Gamma}(a \mid y)= \\
& \frac{(\kappa+1)}{e^{\kappa}} \sum_{t=0}^{\infty} \frac{\kappa(\kappa+1)}{(t !)^{2}} \int_{\Psi}^{\infty} \exp [-(\kappa+1) a / y](a / y)^{t} \frac{d a}{y}= \\
& =\exp [-(\kappa+1) \Psi / y]\left[e^{-\kappa} \sum_{t=0}^{\infty} \frac{\kappa^{t}}{t !} \sum_{\ell=0}^{t} \frac{[(\kappa+1) \Psi]^{\ell}}{\ell ! y^{\ell}}\right]
\end{aligned}
$$

In this case, we have:

$$
\mathbb{E}\left[R^{2}\right]=\int_{0}^{\infty} 2 \rho d \rho P_{S \mid \Gamma}\left(\frac{\mathrm{K} \rho^{-\alpha} P_{t x}}{W}\right),
$$

which by virtue of (20), yields to

$$
\mathbb{E}\left[R^{2}\right]=\left(\frac{2}{\alpha}\right)\left(\frac{\mathrm{K} P_{t x}}{\Psi W(\kappa+1)}\right)^{\frac{\alpha}{2}} e^{-\kappa} \sum_{t=0}^{\infty} \frac{\kappa^{t}}{t !} \sum_{\ell=0}^{t} \frac{\Gamma\left(\frac{2}{\alpha}+\ell\right)}{\ell !}
$$

and finally to the node isolation probability:

$$
\begin{aligned}
& P_{I}=\exp \left\{-\lambda \pi\left(\frac{2}{\alpha}\right)\left(\frac{\mathrm{K} P_{t x}}{\Psi W(\kappa+1)}\right)^{\frac{\alpha}{2}}\right. \\
& \left.e^{-\kappa} \sum_{t=0}^{\infty} \frac{\kappa^{t}}{t !} \sum_{\ell=0}^{t} \frac{\Gamma\left(\frac{2}{\alpha}+\ell\right)}{\ell !}\right\} .
\end{aligned}
$$

The analysis can also be extended to the case of superimposed lognormal shadowing and Rician fading, where:

$$
\begin{aligned}
P_{I}=\exp \left\{-\lambda \pi \int_{0}^{\infty} \frac{d a}{\sqrt{2 \pi} \sigma a} \int_{0}^{\infty} 2 \rho d \rho .\right. \\
\left.\cdot \exp \left[-\frac{1}{2}\left(\frac{\ln \left(\frac{a \rho^{\alpha}}{\mathrm{K}}\right)}{\sigma}\right)^{2}\right] P_{S \mid \Gamma}\left(\frac{a P_{t x}}{W}\right)\right\} .
\end{aligned}
$$

We can thus proceed further in the evaluation of the isolation probability as for the Rayleigh case, noticing that [36, Formula 3.478.1]

$$
\int_{0}^{\infty} x^{\nu-1} \exp \left(-\mu x^{p}\right) d x=\frac{\mu^{-\nu / p}}{|p|} \Gamma\left(\frac{\nu}{p}\right) .
$$

Combining with (16), the final result of the integration is

$$
\left(\frac{2}{\alpha}\right)\left(\frac{K P_{t x}}{\Psi W(\kappa+1)}\right)^{\frac{\alpha}{2}} e^{\left(\frac{\sqrt{2} \sigma}{\alpha}\right)^{2}} e^{-\kappa} \sum_{t=0}^{\infty} \frac{\kappa^{t}}{t !} \sum_{\ell=0}^{t} \frac{\Gamma\left(\frac{2}{\alpha}+\ell\right)}{\ell !}
$$

and finally the node isolation probability is

$$
\begin{aligned}
& P_{I}=\exp \left\{-\lambda \pi\left(\frac{2}{\alpha}\right)\left(\frac{\mathrm{K} P_{t x}}{\Psi W(\kappa+1)}\right)^{\frac{\alpha}{2}} e^{\left(\frac{\sqrt{2} \sigma}{\alpha}\right)^{2}} .\right. \\
& \left.\cdot e^{-\kappa} \sum_{t=0}^{\infty} \frac{\kappa^{t}}{t !} \sum_{\ell=0}^{t} \frac{\Gamma\left(\frac{2}{\alpha}+\ell\right)}{\ell !}\right\}
\end{aligned}
$$

which for $\kappa=0$ reduces to (15) as expected.

A natural way to establish a stochastic ordering is in this case to look at the influence of the $\kappa$ factor on the node isolation probability. By inspection of (21) we see in fact that $\mathbb{E}\left[R_{\kappa_{1}}^{2}\right] \leq \mathbb{E}\left[R_{\kappa_{2}}^{2}\right]$ when $\kappa_{1} \geq \kappa_{2}$, hence the node isolation probability decreases with increasing values of $\kappa$, as expected. In Fig.5, still referring to the same parameters set as Fig. 3, the node isolation probability is plotted vs. the node density both for the cases of superimposed Rice fading and lognormal shadowing, and the case of Rice fading without shadowing. Notice that the beneficial impact of the shadowing is further confirmed, while the node isolation probability increases very slowly with $\kappa$.

\section{G. Random Transmission Power Selection}

The main result we can draw from (6) is that any mechanism able to increase the second-order moment of the transmission range enhances the network performance. Since the mean value of the transmission range is determined by physical layer constraints (noise, average transmission power, SNR threshold and path loss factor), it seems quite natural to look for a technique able to enhance the variance of the transmission range. In this view, the easiest thing to do would be to allow the terminals to independently randomly choose a power level 


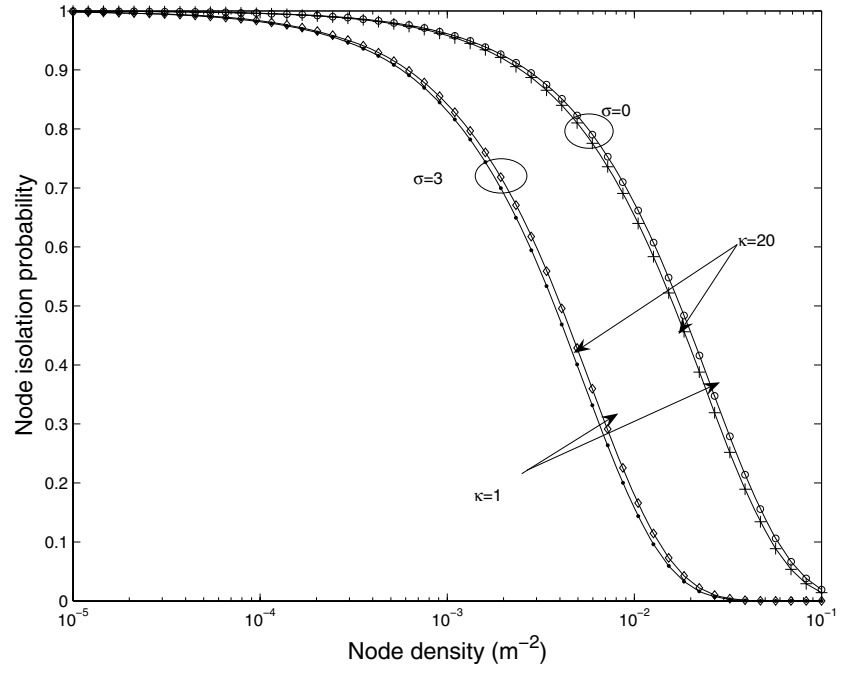

Fig. 5. Node isolation probability vs. node density: the impact of Rice fading; $P_{t x}=1 \mathrm{mWatt}, K=10, W=0.01 \mathrm{mWatt}, \Psi=10 \mathrm{~dB}, \alpha=3.5$, $\sigma=0,3, \kappa=1,20$.

$P_{t x}$ according to a distribution $F_{P_{t x}}(\cdot)$ on which we pose only a constraint on the mean value, namely:

$$
\mathbb{E}\left[P_{t x}\right]=\int_{0}^{+\infty} \mathrm{d} a\left[1-F_{P_{t x}}(a)\right]=\omega .
$$

We get the following proposition, whose proof can be found in [12]:

Proposition 1: Given an ad hoc network where nodes are distributed according to a Poisson point process of intensity $\lambda$, and in the presence of a deterministic channel model/lognormal shadowing/superimposed Rayleigh fading and lognormal shadowing, under the constraint (23), it is not possible to reduce the node isolation probability by means of any mechanism which randomly selects the transmission power.

\section{Connectivity Analysis And Results}

\section{A. Asymptotic Connectivity}

One of the most important issues in ad hoc networks is that of connectivity. Indeed, the conventional paradigms based on end-to-end communications require, as a preliminary condition, the network to be fully connected. ${ }^{6}$

In this section, we aim at characterizing the probability that the nodes in a given bounded, convex subarea of the plane form a connected topology. In general, this question cannot be answered exactly (see [24] for more details). However, we may still get some insight taking the intensity of our driving process $\mathcal{N}$ to infinity (i.e., considering a "very dense" network) and scaling the communication range in such a way that the probability of a node being isolated vanishes. In such a situation, the probability of the network being connected tends to the probability that no isolated nodes are present. This approximation, which clearly offers an optimistic bound

\footnotetext{
${ }^{6}$ Note that this contrasts with the approach followed in the flourishing area of delay-tolerant networking, where communication is based on opportunistic forwarding [37]
}

on the probability of the network to be connected, has been shown in [10] to provide good results. Furthermore, such result is known to hold for the case of deterministic channel model [7], [30]. Consider a bounded, convex set $D$ of area $\mu$. The number of points in $D$ forms a Poisson r.v. with intensity $\lambda \mu$. Let us introduce the following approximations:

(i) the region $D$ is sufficiently large, so that border effects may be neglected [35];

(ii) the probability that a node is isolated, given that there are $n$ nodes in $D$, is given by $P_{I}$;

(iii) given $n$ nodes in $D$, the events $I_{k}=$ \{node $k$ is isolated $, k=1, \ldots, n$ are independent.

As a consequence, the probability of the event \{absence of isolated nodes in $D \mid n$ nodes are present $\}$ is given by $\left(1-P_{I}\right)^{n}$. Using the total probability theorem, we have:

$$
\mathbb{P}[A]=\sum_{n=0}^{\infty} e^{-\lambda \mu} \frac{\left[\lambda \mu\left(1-P_{I}\right)\right]^{n}}{n !}=e^{-\lambda \mu P_{I}} .
$$

where $A$ is the event $\{$ absence of isolated nodes\}. Notice that the approximations introduced above, while clearly representing a simplistic picture of the network behavior, have proven in [10] to provide a satisfactory level of approximation. We conjecture that, even relaxing independence assumption between the node isolation events, still a similar result can be proved, relying on the basis of Chen-Stein method (see, e.g. [38]).

The next step is to approximate (in the limiting regime) the event $C=\{$ the network is connected $\}$ with the event $A$ (absence of isolated nodes). This means that the network would get connected at the same moment at which the last isolated node becomes able to communicate successfully with another node. While there is no proof that this holds in general, such result apply rigorously to the case of both deterministic channel (geometric random graphs [30]) as well as pure ErdösRényi random graphs [29].

The node isolation probability $P_{I}$ is a function of $P_{t x}$; we shall allow $P_{t x}$ to be a function of $\lambda$ as well. From (24), the dependence of $\mathbb{P}[A]$ on $\lambda$ is through the behavior of $\lambda P_{I}$.

We shall say that the network is asymptotically connected if $\lim _{\lambda \rightarrow+\infty} \mathbb{P}[A]=1$. Under the approximations $(i)-(i i i)$, from (24) a necessary and sufficient condition for asymptotic connectivity of any finite $D$ is:

$$
\lim _{\lambda \rightarrow \infty} \lambda P_{I}=0 .
$$

Let us assume that we are in the presence of a deterministic channel model/lognormal shadowing/superimposed Rayleigh fading and lognormal shadowing, and let the transmission power scale as:

$$
P_{t x}=\left(\frac{1}{\pi \xi} \cdot \frac{\ln \lambda+c(\lambda)}{\lambda}\right)^{\frac{\alpha}{2}},
$$

where the expression of $\xi$ depends on the channel model (see $\S$ III-G). For the three channel models considered, we have $P_{I}=e^{-\lambda \pi \xi\left(P_{t x}\right)^{\frac{2}{\alpha}}}$. Using the expression (26), we have:

$$
P_{I}=e^{-(\ln \lambda+c(\lambda))}=\frac{e^{-c(\lambda)}}{\lambda} .
$$




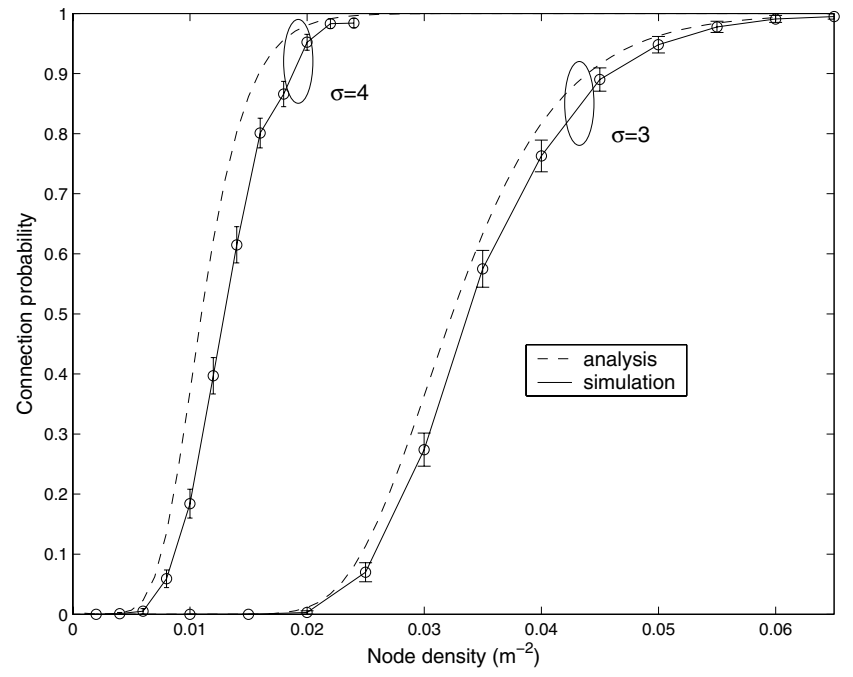

Fig. 6. Connection probability vs. node density in the presence of lognormal shadowing; $P_{t x}=10 \mathrm{mWatt}, K=10, W=0.01 \mathrm{mWatt}, \Psi=10 \mathrm{~dB}$, $\alpha=3.5, \sigma=3,4$.

From (25), a necessary and sufficient condition for asymptotic connectivity is $e^{-c(\lambda)} \rightarrow 0$, from which a necessary and sufficient condition for asymptotic connectivity is:

$$
\lim _{\lambda \longrightarrow \infty} c(\lambda)=+\infty .
$$

It is worth noting that the scaling law of the transmission power for asymptotic connectivity in dense networks is insensitive to the presence of shadowing and/or fading phenomena (up to a multiplicative factor). This insensitivity is really important since it provides guidelines for a robust dimensioning of the network. Otherwise stated, the planning of an ad hoc network can be made relying on a simplistic deterministic channel model. On the other hand, a factor of great importance is the path loss factor $\alpha$, so that a conservative value of such parameter should be considered in the dimensioning phase.

In order to check for the validity of relationship $\mathbb{P}[A] \approx$ $\mathbb{P}[C]$, which has been derived under some simplifying approximations, we simulated a $100 \mathrm{~m} \times 100 \mathrm{~m}$ network, with the same parameters of $\S$ III-D but with a transmission power of 10 mWatt. Simulations were run for $\sigma=3,4$ and, in order to overcome the border effect, a toroidal distance metric was used [35]. The results, averaged over 1000 simulations, are shown in Fig. 6 in terms of $95 \%$ confidence interval. As it may be seen, the simulation results closely follow the predicted ones for both cases, slightly underestimating the network connection probability.

\section{B. Connectivity for Networks with Unreliable Devices}

The asymptotic analysis can be easily extended to the case of unreliable devices [39], [40], where each device is assumed to be active with probability $p$. We let $p$ be a function of $\lambda$ (which allows for an engineering tradeoff between devices reliability and nodes density), and study scaling laws for the transmission power. Let the channel be characterized by a path loss factor $\alpha$, regardless of the possible presence of shadowing and/or Rayleigh fading and let $P_{t x}(\lambda)$ denote the transmission power. Then, under approximations $(i)-($ iii $)$, and threading the footprints of [39], [40], it is easy to show that the followings hold:

(a) a necessary and sufficient condition for asymptotic connectivity is given by:

$$
\lim _{\lambda \rightarrow+\infty} \lambda p(\lambda) e^{-\lambda p(\lambda)\left(P_{t x}(\lambda)\right)^{\frac{2}{\alpha}}}=0 .
$$

(b) If $p=\lim _{\lambda \rightarrow+\infty} p(\lambda)$ satisfies $0<p<1$ and $P_{t x}(\lambda)=$ $\left(\frac{1}{\pi \xi} \cdot \frac{\ln \lambda+c(\lambda)}{\lambda}\right)^{\frac{\alpha}{2}}$, the network is asymptotically connected at distance $d$ if $c(\lambda)$ satisfies

$$
c(\lambda) \geq \zeta \ln \lambda
$$

where $\zeta>\frac{1-p}{p}$. Furthermore, the network is asymptotically connected only if $c=\lim _{\lambda \rightarrow+\infty} c(\lambda)=+\infty$.

(c) Let $p(\lambda)$ be $p(\lambda)=\lambda^{-\gamma}$, where $0<\gamma<1$. Then a sufficient condition for asymptotic network connectivity at distance $d$ is given by:

$$
p(\lambda)\left[P_{t x}(\lambda)\right]^{\frac{2}{\alpha}} \geq \zeta \frac{\ln \lambda}{\lambda},
$$

where the constant $\zeta$ satisfies $\zeta>1-\gamma$.

\section{Exploiting Diversity to ENHANCE Network CONNECTIVITY}

In this section we explore the possibility of improving the connectivity of the network in the presence of superimposed Rayleigh fading and lognormal shadowing by using multiple antennas at the receiver side. Apart from the theoretical importance of the issue, it is worth noting that diversity can be exploited not only by using multiple antennas, but also by means of cooperation schemes [41], [42] and time diversity techniques, where the spatial dimension of the network itself and, respectively, the time varying nature of the channel, is used to provide diversity.

Assuming that $M$ antennas are present at the receiver, and that they are spaced apart, so that the paths from the transmitter to the receiver antennas may be considered independent, we study the impact of two signal processing technique, namely:

- best path selection (BPS): among the $M$ received signals, the one with the largest SNR is decoded;

- maximal ratio combining (MRC): the $M$ received signals are combined in such a way to maximize the overall SNR (see [20] for more details).

Let us focus on BPS; given an average SNR of $\Gamma=y$, the probability that at least one path presents a SNR greater than $\Psi$ is the complement of the probability of all paths presenting an SNR lower than $\Psi$ :

$$
\begin{aligned}
P_{S \mid \Gamma}(y)=1-\prod_{k=1}^{M} \int_{0}^{\Psi} \mathrm{d} a_{i} f_{\gamma_{i} \mid \Gamma}\left(a_{i} \mid y\right)= \\
=1-\left[\int_{0}^{\Psi} \mathrm{d} a \frac{1}{y} e^{-\frac{a}{y}}\right]^{M}=1-\left(1-e^{-\frac{\Psi}{y}}\right)^{M}= \\
=-\sum_{h=1}^{M}\left(\begin{array}{c}
M \\
h
\end{array}\right)(-1)^{h} e^{-\frac{h \Psi}{y}} .
\end{aligned}
$$


Substituting (31) in (6), together with (8), we get:

$$
\begin{gathered}
P_{I}=\exp \left\{\lambda \pi \sum_{h=1}^{M}\left(\begin{array}{c}
M \\
h
\end{array}\right)(-1)^{h} .\right. \\
\cdot\left[\int_{0}^{+\infty} \mathrm{d} a \int_{0}^{+\infty} \mathrm{d} \rho e^{-\frac{h \Psi W}{a P_{t x}}} 2 \rho \frac{1}{\sqrt{2 \pi} \sigma a} e^{\left.\left.-\frac{1}{2}\left(\frac{\ln \frac{a \rho^{\alpha}}{K}}{\sigma}\right)^{2}\right]\right\}=}\right. \\
=\exp \left\{\lambda \pi \sum_{h=1}^{M}\left(\begin{array}{c}
M \\
h
\end{array}\right)(-1)^{h} .\right. \\
\left.\cdot\left(\int_{-\infty}^{+\infty} \mathrm{d} x \int_{0}^{+\infty} \mathrm{d} \rho \frac{1}{\sqrt{2 \pi}} e^{-\frac{x^{2}}{2}} 2 \rho e^{-\frac{h \Psi W e^{-\sigma x} \rho^{\alpha}}{K P_{t x}}}\right)\right\} .
\end{gathered}
$$

Some cumbersome algebra leads to :

$$
\begin{array}{r}
P_{I}=\exp \left\{\lambda \pi \frac{2}{\alpha} \Gamma\left(\frac{2}{\alpha}\right)\left(\frac{\Psi W}{K P_{t x}}\right)^{-\frac{2}{\alpha}} e^{\left(\frac{\sqrt{2} \sigma}{\alpha}\right)^{2} .}\right. \\
\left.\cdot \sum_{h=1}^{M}\left(\begin{array}{c}
M \\
h
\end{array}\right)(-1)^{h} h^{-\frac{2}{\alpha}}\right\} .
\end{array}
$$

Since

$$
\sum_{h=1}^{M}\left(\begin{array}{c}
M \\
h
\end{array}\right)(-1)^{h} h^{-\frac{2}{\alpha}} \leq-1,
$$

we may conclude that the use of receiver diversity with best path selection improves the connectivity properties of the resulting network, as expected. Note, however, that the scaling for asymptotic connectivity remains unchanged.

In case of MRC, the system behaves as if the channel consisted of an $M$-Nakagami fading superimposed to lognormal shadowing [20]. The pdf of the instantaneous SNR is given by:

$$
f_{\gamma \mid \Gamma}(a \mid y)=\left(\frac{M}{y}\right)^{M} \frac{a^{M-1}}{\Gamma(M)} e^{-M \frac{a}{y}}
$$

where $\Gamma(\cdot)$ is the Gamma function. Note that for $M=1$ we obtain the usual expression for Rayleigh fading. The ccdf is given by:

$$
P_{S \mid \Gamma}(y)=\mathbb{P}[\gamma>\Psi \mid \Gamma=y]=1-F_{\gamma}(\Psi)=\frac{\Gamma\left(M, \frac{M \Psi}{y}\right)}{\Gamma(M)},
$$

where $\Gamma(\cdot, \cdot)$ is the incomplete Gamma function [36]. Since $M$ is an integer, the expression above simplifies to:

$$
P_{S \mid \Gamma}(y)=e^{-M \frac{\Psi}{y}} \sum_{h=0}^{M-1}\left(\frac{M \Psi}{y}\right)^{h} \frac{1}{(h+1) !} .
$$

Proceeding as above, the node isolation probability turns out to be:

$$
\begin{gathered}
P_{I}=\exp \left\{-\lambda \pi \sum_{h=0}^{M-1} \frac{1}{(h+1) !}\left[\int_{0}^{+\infty} \mathrm{d} a \int_{0}^{+\infty} \mathrm{d} \rho e^{-\frac{\Psi W}{a P_{t x}} .}\right.\right. \\
\left.\left.\cdot\left(\frac{M \Psi W}{a P_{t x}}\right)^{h} 2 \rho \frac{1}{\sqrt{2 \pi} \sigma a} e^{-\frac{1}{2}\left(\frac{\ln a-\ln \left(K \rho^{-\alpha}\right)}{\sigma}\right)^{2}}\right]\right\},
\end{gathered}
$$

that after some algebra reduces to:

$$
\begin{aligned}
P_{I}=\exp \left\{-\lambda \pi \frac{2}{\alpha} e^{\left(\frac{\sqrt{2} \sigma}{\alpha}\right)^{2}}\left(\frac{\Psi W}{K P_{t x}}\right)^{-\frac{2}{\alpha}} .\right. \\
\left.\cdot \sum_{h=0}^{M-1} \frac{1}{(h+1) !} M^{h} \Gamma\left(h+\frac{2}{\alpha}\right)\right\} .
\end{aligned}
$$

Since

$$
\sum_{h=0}^{M-1} \frac{1}{(h+1) !} M^{h} \Gamma\left(h+\frac{2}{\alpha}\right) \geq \Gamma\left(\frac{2}{\alpha}\right),
$$

also receiver-based diversity with MRC improves the network connectivity.

For both BPS and MRC, the connectivity improvement could be predicted by establishing a stochastic ordering among the distributions of the communication range $R$ in the presence of different number of antennas. It suffices to note that for both schemes, if $M_{1} \leq M_{2}, P_{S \mid \Gamma, M_{1}} \leq P_{S \mid \Gamma, M_{2}}$, where $P_{S \mid \Gamma, x}$ is the probability of successful reception given an average SNR of $\Gamma$ and in the presence of $x$ antennas. Substituting in (5), it is easy to see that a stochastic ordering among the distribution corresponding to different values of $M$ is possible:

$$
R_{M_{1}} \preceq R_{M_{2}} \quad M_{1} \leq M_{2},
$$

and the connectivity improvement follows along the arguments outlined in $\S$ III-D.

\section{A. A Special Case: MIMO MRC}

We now turn our attention to the case of MIMO processing, where multiple antennas are employed at both the transmitter and the receiver side. We assume that $M$ antennas are present at the transmitter end and at the receiver one. We assume to employ MRC at the receiver to deal with simple instantaneous SNR distribution expressions, nevertheless our results hold for any informed transmitter communication scheme, as discussed in [43]. For the sake of simplicity, we focus on a MIMO Rayleigh faded channel, whose antennas are assumed to be uncorrelated at both transmitter and receiver ends. The probability of successful reception can be written as [44] $P_{S \mid \Gamma}(y)=\beta \operatorname{det}(\Pi)$, where $\Pi$ is matrix with $(i, j)$-th entry $\Pi_{i, j}=\gamma(i+j-1, a / y), \gamma(\cdot, \cdot)$ is the lower incomplete gamma function [45] and $\beta=\left[\prod_{\ell=1}^{M}(M-\ell) !^{2}\right]^{-1}$. In order to get relatively concise expressions from where to provide connectivity guidelines we assume $M=2$; however, the derivation can be repeated for any $M$ always getting closedform results

Now, recasting the expression of $P_{S \mid \Gamma}(y)$ using [46, Formula (23)] and some algebra, and still exploiting (20), we can finally write the node isolation probability as

$$
\begin{array}{r}
P_{I}=\exp \left\{-\lambda \pi\left(\frac{2}{\alpha}\right) \Gamma\left(\frac{2}{\alpha}\right)\left(\frac{K P_{t x}}{\Psi W}\right)^{\frac{\alpha}{2}} e^{\left(\frac{\sqrt{2} \sigma}{\alpha}\right)^{2}} \beta .\right. \\
\left.\cdot\left[\left(\frac{2}{\alpha}\right)\left(\frac{2}{\alpha}+1\right)+2-2^{-\frac{2}{\alpha}}\right]\right\},
\end{array}
$$

where one can see immediately the gain in terms of $\mathbb{E}\left[R^{2}\right]$ achievable through the use of nodes equipped with two antennas instead of a single one. This gain in terms of node 


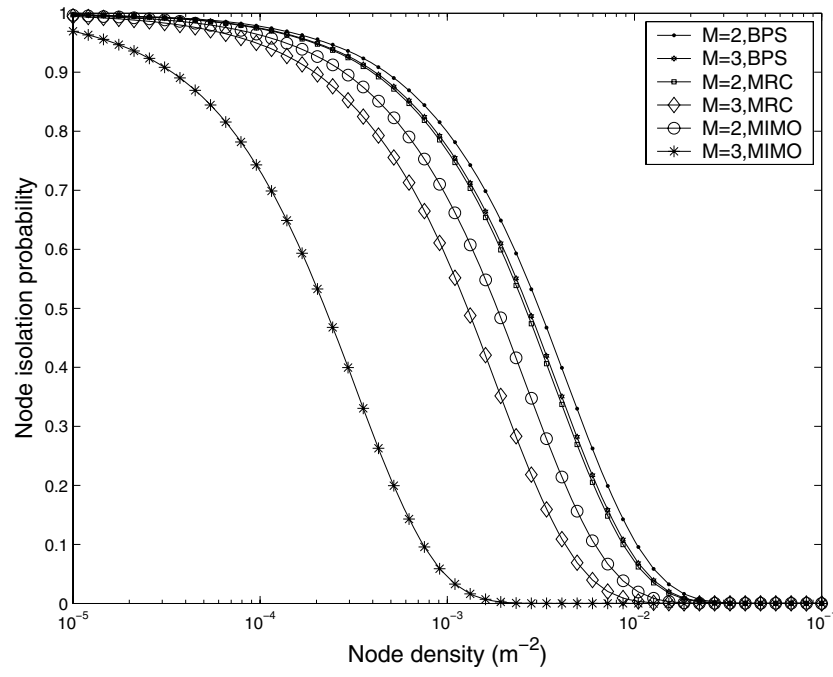

Fig. 7. Node isolation probability vs. node density: comparison of best path selection (BPS), maximal ratio combining (MRC) and MIMO MRC; $P_{t x}=1$ mWatt, $K=10, W=0.01$ mWatt, $\Psi=10 \mathrm{~dB}, \alpha=3.5, \sigma=3$.

isolation probability still holds for increasing values of $M$. In particular, even if a general formula cannot be provided for generic values of $M$, due to the particular expression of $P_{S \mid \Gamma}(y)$ involving a determinant, $P_{I}$ can be anyway always evaluated in closed form for fixed $M$, under Rayleigh fading conditions, even for the case of correlated links, always starting from the results in [46]. A direct comparison of the performance achieved by the three different transmit and/or receive diversity schemes is represented in Fig. 7, where it may be seen that MRC outperforms the simpler BPS scheme, and that the performance achievable with BPS and $M=3$ antennas are slightly worse than those achievable by limiting the number of antennas to $M=2$ and employing a MRC scheme. Furthermore, the beneficial impact of the deployment of multiple antennas both at the transmitter as well as at the receiver is confirmed by drawing the node isolation probability both for the case of $M=2$ and $M=3$ transmit and receive antennas. Notice finally that we assume all nodes to have the same number of antennas only for symmetry reasons, but such an assumption can be relaxed without loss of generality and still the node isolation probability can be evaluated in closed form.

\section{CONCLUSIONS}

In this paper we have presented an analytical procedure for the computation of the node isolation probability in an ad hoc network in the presence of channel randomness, with a particular emphasis on the effect of lognormal shadowing, Rayleigh and Rice fading phenomena. We have shown that the node isolation probability decreases as the lognormal spread $\sigma$ increases, and investigated the negative effect of both analyzed fading models. We proved that no connectivity improvement may be achieved, under an average power constraint, by means of transmission power random selection schemes. The coverage probability coincides with the complement of the node isolation probability computed under an equivalent communication range distributed as the sensing range, given that nodes are distributed according to a Poisson point process; otherwise it is possible to relate the two quantities through an inversion formula.

From the node isolation probability we can get, under some simplifying approximations, a closed form formula for the probability of the network being connected in the presence of a very large device density. Simulation results have been presented, which show the soundness of the proposed procedure. For the case of superimposed shadowing and Rayleigh fading, the connectivity improvement achievable by means of two simple diversity schemes, best path selection and maximal ratio combining, has been investigated; a general stochastic ordering relationship has been found for both schemes, showing the beneficial impact of the use of diversity schemes; the investigation has been also extended to MIMO maximal ratio combining technique, showing the advantages achievable with respect to the case where multiple antennas are used at just one of the communication end points.

Directions for future work include the generalization of the results to more general node placement distributions and a study of the impact of various mobility patterns on the connectivity results found for a static scenario.

\section{REFERENCES}

[1] I. F. Akyildiz, W. Su, Y. Sankarasubramaniam, and E. Cayirci, "Wireless sensor networks: A survey," Computer Networks, vol. 38, no. 4, pp. 393422, Mar. 2002.

[2] P. Gupta and P. R. Kumar, "Critical power for asymptotic connectivity in wireless networks," in Stochastic Analysis, Control, Optimization and Applications: A Volume in Honor of W. H. Fleming. Boston: Birkh'auser, 1998.

[3] _ - "The capacity of wireless networks," IEEE Trans. Inform. Theory, vol. 46, no. 2, pp. 388-404, Mar. 2000.

[4] A. E. Gamal, J. Mammen, B. Prabhakar, and D. Shah, "Throughputdelay trade-off in wireless networks," in Proc. IEEE INFOCOM, vol. 1, Hong Kong, 2004.

[5] J. Orriss and S. K. Barton, "Probability distributions for the number of radio transceivers which can communicate with one another," IEEE Trans. Commun., vol. 51, no. 4, pp. 676-681, Apr. 2003.

[6] P. Gupta and P. R. Kumar, "Critical power for asymptotic connectivity," in Proc. IEEE CDC, Tampa, FL, 1998, pp. 1106-1110.

[7] M. D. Penrose, "The longest edge of the random minimal spanning tree," The Annals of Appl. Prob., vol. 7, no. 2, pp. 340-361, 1997.

[8] P. Santi and D. M. Blough, "The critical transmitting range for connectivity in sparse wireless ad hoc networks," IEEE Trans. Mobile Computing, vol. 2, no. 1, pp. 25-39, Jan.-Mar. 2003.

[9] P. Santi, "The critical transmitting range for connectivity in mobile ad hoc networks," IEEE Trans. Mobile Computing, vol. 4, no. 3, pp. 310317, May/June 2005.

[10] C. Bettstetter and C. Hartmann, "Connectivity of wireless multihop networks in a shadow fading environment," Wireless Networks, vol. 11, no. 5, pp. 571-579, May 2005.

[11] R. Hekmat and P. Van Mieghem, "Connectivity in wireless ad-hoc networks with a log-normal radio model," Mobile Networks and Applications, vol. 11, pp. 351-360, June 2006.

[12] E. Altman and D. Miorandi, "Coverage and connectivity of ad-hoc networks in presence of channel randomness," in Proc. IEEE INFOCOM, Miami, FL, 2005, pp. 491-502.

[13] J. Orriss and S. K. Barton, "A statistical model for connectivity between mobiles and base stations: the extension to Suzuki," 2002, COST 273 $\mathrm{TD}(02) 121$.

[14] _ "A statistical model for connectivity between mobiles and base stations: from Suzuki to Rice and beyond," 2003, COST 273 TD(03) 89.

[15] M. Haenggi, "A geometry-inclusive fading model for random wireless networks," in Proc. IEEE ISIT, Seattle, WA, 2006, pp. 1329-1333.

[16] O. Dousse, F. Baccelli, and P. Thiran, "Impact of interferences on connectivity in ad-hoc networks," IEEE/ACM Trans. Networking, vol. 13, no. 2, pp. 425-436, July 2005. 
[17] F. Baccelli and B. Blaszczyszyn, "On a coverage process ranging from the Boolean model to the Poisson Voronoi tessellation with applications to wireless communications," INRIA, Tech. Rep. 4019, Oct. 2000.

[18] O. Dousse and P. Thiran, "Connectivity vs capacity in dense ad hoc networks," in Proc. IEEE INFOCOM, vol. 1, Hong Kong, 2004.

[19] T. S. Rappaport, Wireless Communications. Upper Saddle River, NJ: Prentice Hall, 1999.

[20] L. Hanzo, C. H. Wong, and M. S. Yee, Adaptive Wireless Transceivers. New York: John Wiley and Sons, 2002.

[21] X.-Y. Li, P.-J. Wan, and O. Frieder, "Coverage in wireless ad hoc sensor networks," IEEE Trans. Computers, vol. 52, no. 6, pp. 753-763, June 2003.

[22] F. Baccelli and P. Bremaud, Elements of Queueing Theory. Berlin: Springer-Verlag, 1994.

[23] D. Stoyan, W. S. Kendall, and J. Mecke, Stochastic Geometry and its Applications. New York: J. Wiley \& Sons, 1987.

[24] P. Hall, Introduction to the Theory of Coverage Processes. New York: J. Wiley \& Sons, 1988.

[25] R. Meester and R. Roy, Continuum Percolation. New York: Cambridge Univ. Press, 1996.

[26] M. Zorzi and S. Pupolin, "Outage probability in multiple access packet radio networks in the presence of fading," IEEE Trans. Veh. Technol., vol. 43, no. 3, pp. 604-610, Aug. 1994.

[27] L. Booth, J. Bruck, M. Cook, and M. Franceschetti, "Ad hoc wireless networks with noisy links," in Proc. IEEE ISIT '03, Yokohama, Japan, 2003, pp. 386-386.

[28] D. Stoyan, Comparison Methods for Queues and Other Stochasti Models. New York: John Wiley \& Sons, 1983.

[29] B. Bollobás, Random Graphs. Cambridge, UK: Cambridge University Press, 2001.

[30] M. D. Penrose, Random Geometric Graphs. Oxford, UK: Oxford University Press, 2003.

[31] C. Bettstetter, "On the connectivity of ad-hoc networks," The Computer Journal, special issue on mobile and pervasive computing, vol. 47, no. 4, pp. 432-447, July 2004.

[32] A. Helmy, "Small worlds in wireless networks," IEEE Commun. Lett., no. 10 , pp. 490-492, Oct. 2003.

[33] C. Bordenave, "Navigation in self organized networks," in Proc. WiOpt, Boston, MA, 2006.

[34] M. Franceschetti, L. Booth, J. Bruck, M. Cook, and R. Meester, "Continuum percolation with unreliable and spread out connections," J. Statistical Physics, vol. 118, no. 3/4, pp. 721-734, Feb. 2005.

[35] C. Bettstetter and O. Krause, "On border effects in modeling and simulation of wireless ad hoc networks," in Proc. IEEE MWCN, Recife, Brazil, 2001, pp. 20-27.

[36] I. S. Gradshteyn and I. M. Ryzhik, Table of Integrals, Series, and Products. Orlando, FL: Academic Press, 1983.

[37] K. Fall, "A delay-tolerant network architecture for challenged Internets," in Proc. ACM SIGCOMM, Karlsruhe, Germany, 2003.

[38] A. D. Barbour, "Topics in poisson approximation," in Stochastic Processes: Theory and Methods, Handbook of Statistics. Amsterdam, Netherlands: Elsevier Science, 2001.

[39] S. Shakkottai, R. Srikant, and N. B. Shroff, "Unreliable sensor grids: coverage, connectivity and diameter,' in Proc. IEEE INFOCOM, San Francisco, CA, 2003, pp. 1073-1083.

[40] D. Miorandi and E. Altman, "Connectivity in ad-hoc networks: a queueing theoretical approach," in Proc. WiOpt, Cambridge, UK, 2004.

[41] L.-L. Xie and P. R. Kumar, "A network information theory for wireless communications: scaling laws and optimal operation," IEEE Trans. Inform. Theory, vol. 50, no. 5, pp. 748-767, May 2004.
[42] J. N. Laneman, D. N. C. Tse, and G. W. Wornell, "Cooperative diversity in wireless networks: efficient protocol and outage behavior," IEEE Trans. Inform. Theory, vol. 50, no. 12, pp. 3062-3080, Dec. 2004.

[43] A. Molisch and M. Win, "MIMO systems with antenna selection: An overview," IEEE Microwave Mag., vol. 5, no. 1, pp. 46-56, Mar. 2004.

[44] M. Kang and M. Alouini, "Largest eigenvalue of complex Wishart matrices and performance analysis of MIMO MRC systems," IEEE J. Sel. Areas Commun., vol. 21, no. 3, pp. 418-423, Mar. 2003.

[45] M. Abramowitz and I. A. Stegun, Handbook of Mathematical Functions with Formulas, Graphs, and Mathematical Tables. New York: Dover Publications, 1995.

[46] A. Zanella, M. Chiani, and M. Z. Win, "Performance of MIMO MRC in correlated Rayleigh fading environments," in Proc. VTC Spring, Stockolm, Sweden, 2005, pp. 1633-1637.

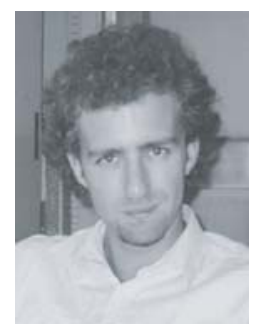

Daniele Miorandi is the head of the Pervasive Area at CREATE-NET, Italy. He received a $\mathrm{PhD}$ in Communications Engineering from Univ. of Padova, Italy, in 2005, and a Laurea degree (summa cum lauda) in Communications Engineering from Univ. of Padova, Italy, in 2001. He joined CREATE-NET in Jan. 2005, where he is leading a group working on Pervasive Computing and Communication Environments. His research interests include bio-inspired approaches to networking and service provisioning in large-scale computing systems, modelling and performance evaluation of wireless networks, wireless extensions of fieldbus systems, prototyping of wireless mesh solutions.

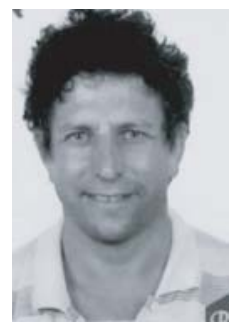

Eitan Altman received the B.Sc. degree in electrical engineering (1984), the B.A. degree in physics (1984) and the Ph.D. degree in electrical engineering (1990), all from the Technion-Israel Institute, Haifa. In (1990) he further received his B.Mus. degree in music composition in Tel-Aviv university. Since 1990, he has been with INRIA (National research institute in informatics and control) in Sophia-Antipolis, France. His current research interests include performance evaluation and control of telecommunication networks and in particular congestion control, wireless communications and networking games. $\mathrm{He}$ is in the editorial board of several scientific journals: Stochastic Models, JEDC, COMNET, SIAM SICON and WINET. He has been the (co)chairman of the program committee of several international conferences and workshops (on game theory, networking games and mobile networks). More information can be found at http://www.inria.fr/mistral/personnel/Eitan.Altman/me.html

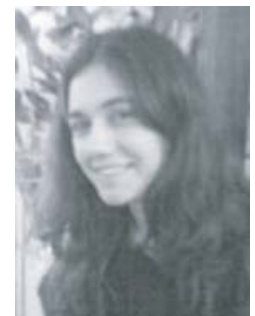

Giuseppa Alfano was born in Naples, Italy, on march 22, 1978. She received Laurea degree in Communication Engineering from University of Naples "Federico II", Italy, in 2001. From 2002 to 2004 she was involved in radar and satellite signal processing studies at National Research Council and University of Naples. Since April 2004 she is ph.d. student in information engineering at University of Benevento, Italy. Her research work lies mainly in the field of finite dimensional random matrix theory with applications to MIMO wireless communica-

tions and sensor networks. 